\title{
Molecular docking of the anticancer bioactive compound proceraside with macromolecules involved in the cell cycle and DNA replication
}

\author{
A.B. Gurung', M.A. Ali ${ }^{2}$, A. Bhattacharjee ${ }^{1}$, M. AbulFarah ${ }^{3}$, F. Al-Hemaid ${ }^{2}$, \\ F.M. Abou-Tarboush ${ }^{3}$, K.M. Al-Anazi ${ }^{3}$, F.S.M. Al-Anazi ${ }^{2}$ and J. Lee ${ }^{4}$ \\ ${ }^{1}$ Department of Biotechnology and Bioinformatics, \\ North Eastern Hill University, Shillong, Meghalaya, India \\ ${ }^{2}$ Department of Botany and Microbiology, College of Science, \\ King Saud University, Riyadh, Saudi Arabia \\ ${ }^{3}$ Department of Zoology, College of Science, King Saud University, \\ Riyadh, Saudi Arabia \\ ${ }^{4}$ Department of Environment and Forest Resources, \\ Chungnam National University, Yuseong-gu, Daejeon, Republic of Korea \\ Corresponding author: M.A. Ali \\ E-mail: alimohammad@ksu.edu.sa
}

Genet. Mol. Res. 15 (2): gmr.15027829

Received October 15, 2015

Accepted December 28, 2015

Published May 6, 2016

DOI http://dx.doi.org/10.4238/gmr.15027829

\begin{abstract}
The bioactive compounds proceraside A, frugoside and calotropin, which were extracted from the root bark of Calotropis procera (Aiton) W.T. Aiton (family Asclepiadaceae), were recently reported to inhibit the growth of inhibition against various human cancer cell lines in vitro. However, their modes of action have not been clearly defined. Therefore, we attempted an in silico approach to gain insights into their binding modes against the following selected molecular targets: CDK-2, CDK-6, topoisomerase I, BCL-2, VEGFR-2, telomere: G-quadruplex, and topoisomerase II. These targets were selected based on their key roles in cancer progression via the regulation of the cell cycle and DNA replication. Molecular-docking analyses revealed that
\end{abstract}


proceraside A was the best docked ligand against all the targets, with the exception of telomere-G: quadruplex. Furthermore, it displayed the lowest binding energies and inhibition constants, and critical hydrogen bonds and hydrophobic interactions with the targets were also revealed. The present study may aid in the identification of possible targets for proceraside $\mathrm{A}$, and might provide a plausible explanation for its proven anti-tumor activities. Moreover, the result of this study may further guide structure-activity relationship studies used to generate more potent target-specific inhibitors.

Key words: Proceraside; Molecular docking; Anticancer; Cell cycle; DNA replication

\section{INTRODUCTION}

Cancer is a major global health problem (Siegel et al., 2015) and is characterized by invasive and uncontrolled cell division and the spread of abnormal cells (Greenlee et al., 2000; Gan et al., 2003). The discovery of cancer treatment drugs is a very complex and costly endeavor, and only a few drugs that undergo clinical trials actually reach patients. Highthroughput screening (HTS) is the primary method used to identify initial lead compounds for a particular target disease. However, HTS has a high failure rate, and it does not efficiently identify desired viable drug leads in most cases. Molecular docking (an alternative HTS method) provides a rapid way to evaluate likely binders from large chemical libraries with minimal costs, and it is being widely used as an important component of the drug discovery process (Stark and Powers, 2012). Recently, the cardiac glycoside proceraside A, together with frugoside and calotropin were isolated from the ethyl acetate fraction of the methanolic extract of the root bark of Calotropis procera (Aiton) W.T. Aiton (family Asclepiadaceae). The compounds were reported to have potent in vitro growth inhibitory activity against human A549 NSCLC, U373 GBM, and PC-3 prostate cancer cells (Ibrahim et al., 2014) but their modes of action have not been clearly defined. Therefore, as a preliminary investigation of the potential molecular targets and to confirm the experimental activity testing for proceraside, molecular docking was performed using the following enzymes involved in cell cycle, cell growth and DNA replication: cyclin-dependent protein kinase 2 (CDK-2), CDK-6, DNA topoisomerases I and II, receptor proteins B-cell lymphoma 2 (BCL-2), vascular endothelial growth factor receptor 2 (VEGFR-2), and telomere:G-quadruplex.

\section{MATERIAL AND METHODS}

\section{Preparation of ligand and receptor}

The structures of three major C. procera compounds (proceraside A, frugoside, and calotropin) were modeled using the Chemsketch software (http://www.acdlabs.com/resources/ freeware/) (Figure 1). The structures were optimized using the MMFF94 force field (Halgren, 1996). The optimization parameters included a number of steps of the 500 steepest descent algorithm and a convergence criterion of $10 \mathrm{e}-7$, and the optimized compounds were used to 
perform molecular docking. The three-dimensional structures of the following seven molecular targets (receptors) were obtained from Protein Data Bank (PDB) (www.rcsb.org): CDK-2, CDK-6, topoisomerase I, BCL-2, VEGFR-2, telomere: G-Quadruplex and topoisomerase II. The steps for receptor preparation included the removal of heteroatoms (water and ions), the addition of polar hydrogen, and the assignment of Kollman charges. The active sites were defined using grid boxes of appropriate sizes around the bound cocrystal ligands as is shown in Table 1.
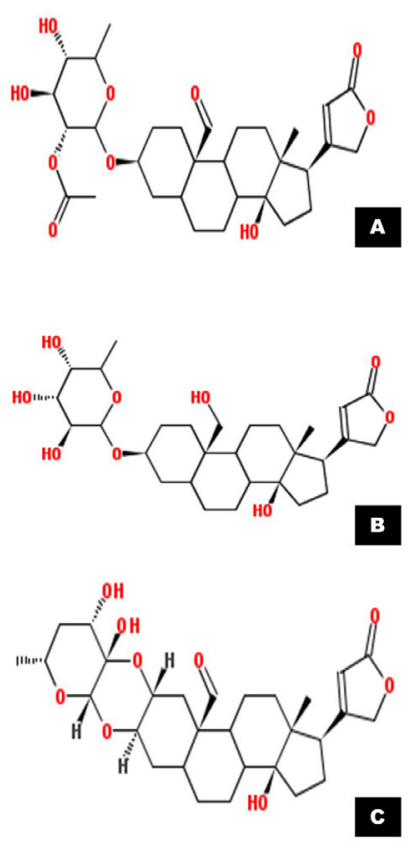

Figure 1. A.-C. Chemical structures of Calotropis procera-derived compounds selected for molecular docking.

Table 1. Protein targets, cocrystal ligand, active site residues, and grid box dimensions.

\begin{tabular}{|c|c|c|c|c|c|}
\hline \multirow{2}{*}{ Protein targets } & \multirow{2}{*}{ Cocrystal ligand } & \multirow{2}{*}{ Active site residue(s) } & \multicolumn{3}{|c|}{ Grid box dimensions } \\
\hline & & & No. of grid points (npts) & $\begin{array}{l}\text { Center (xyz coordinates) } \\
\end{array}$ & Grid point spacing $(\AA)$ \\
\hline $1 \mathrm{di} 8$ & $\begin{array}{l}\text { 4-[3-Hydroxyanilino]-6,7- } \\
\text { Dimethoxyquinazoline }\end{array}$ & $\begin{array}{l}\text { ILE10(A), VAL18(A), ALA31(A), LYS33(A), PHE80(A), } \\
\text { GLU1(A), PHE82(A), LEU83(A), HIIS84(A), , } 6 \text { (A)85(A), } \\
\text { ASP86(A), LEU134(A), ALA144(A), ASP145(A) }\end{array}$ & $60 \times 60 \times 60$ & $-7.623,49.881,11.367$ & \\
\hline $1 \times 02$ & 3,7,3',4'-Tetrahydroxyflavone & $\begin{array}{l}\text { ILE19(B), ALA41(B), LYS43(B), GLU61(B), PHE98(B), } \\
\text { GLU99(B), HIS100(B), VAL101(B), ASP 104(B), } \\
\text { GLN149(B), LEU152(B), ASP163(B) }\end{array}$ & $60 \times 60 \times 60$ & $2.296,36.095,138.519$ & 0.375 \\
\hline $1 \mathrm{zxm}$ & $\begin{array}{l}\text { Phosphoaminophosphonic Acid-Adenylate } \\
\text { Ester }\end{array}$ & $\begin{array}{l}\text { ASN91(A), ASN95(A), ARG98(A), ASN120(A), } \\
\text { ILE125(A), ILE141(A), PHE142(A), SER148(A), } \\
\text { SER149(A), ASN163(A), GLY164(A), TYR165(A), } \\
\text { GLY166(A), ALA167(A), LYS168(A), THR215(A), } \\
\text { GLN376(A), LYS378(A) }\end{array}$ & $60 \times 60 \times 60$ & $39.262,-1.072,37.077$ & 0.375 \\
\hline $202 \mathrm{f}$ & $\begin{array}{l}\text { 4-(4-Benzyl-4-Methoxypiperidin-1-YI)-N- } \\
\text { [(4-\{[1,1-Dimethyl-2- } \\
\text { (Phenylthio)Ethyl]Amino\}-3- } \\
\text { Nitrophenyl)Sulfonyl]Benzamide }\end{array}$ & $\begin{array}{l}\text { ALA97(A), ASP100(A), PHE101(A), TYR105(A), } \\
\text { ASP108(A), PHE109(A), MET112(A), VAL130(A), } \\
\text { LEU134(A), TRP114(A), GLY142(A), , RRG143(A), } \\
\text { VAL145(A), ALA146(A), PHE150(A), TYR199(A) }\end{array}$ & $65 \times 65 \times 65$ & $-0.024,3.142,-0.361$ & 0.375 \\
\hline $2 \mathrm{oh} 4$ & $\begin{array}{l}\text { Methyl (5-\{4-[(\{[2-Fluoro-5- } \\
\text { (Trifluoromethyl)Phenyl]Amino\} Carbonyl) } \\
\text { Amino]Phenoxy\}-1h-Benzimidazol-2- } \\
\text { Yl)Carbamate }\end{array}$ & $\begin{array}{l}\text { LEU838(A), VAL846(A), ALA864(A), GLU883(A), } \\
\text { LEU887(A), VAL897(A), VAL914(A), GLU915(A), } \\
\text { PHE916(A), CYS917(A), LYS918(A), GLY920(A), } \\
\text { LEU1033(A), CYS1043(A), ASP1044(A), PHE1045(A) }\end{array}$ & $70 \times 70 \times 70$ & $5.396,32.493,15.884$ & 0.375 \\
\hline $111 \mathrm{~h}$ & $\begin{array}{l}\text { 3-Pyrrolidin-1-Y1-N-[6-(3-Pyrrolidin-1-Yl- } \\
\text { Propionylamino)-Acridin-3-YI]- } \\
\text { Propionamide }\end{array}$ & $\begin{array}{l}\text { DT1006(A), DT1007(A), DT1008(A), DG1009(A), } \\
\text { DG2001(B), DT2012(B) }\end{array}$ & $45 \times 45 \times 60$ & $15.456,16.903,7.206$ & 0.375 \\
\hline $1+8 \mathrm{i}$ & $\begin{array}{l}\text { 4-Ethyl-4-Hydroxy-1,12-Dihydro-4h-2- } \\
\text { Oxa-6,12a-Diaza-Dibenzo[B,H]Fluorene- } \\
\text { 3,13-Dione }\end{array}$ & $\begin{array}{l}\text { ARG364(A), THR718(A), ASN722(A), DT10(B), } \\
\text { TGP11(C), DG12(C), DC112(D), DA113(D) }\end{array}$ & $70 \times 70 \times 70$ & $21.171,-3.904,25.952$ & 0.375 \\
\hline
\end{tabular}




\section{Molecular docking}

The proceraside A, frugoside and calotropin compounds were docked against the seven molecular targets using the AutoDock4.2 software (Morris et al., 2009). Docking to molecular targets was executed using the Lamarckian Genetic Algorithm using the following parameters: an initial population of 150 randomly placed individuals; a maximum number of 2,500,000 energy evaluations; a mutation rate of 0.02 ; and a crossover rate of 0.8 . Fifty independent docking runs were performed for each ligand. Conformations that differed by $<2.0 \AA$ root mean square deviations (RMSD) were clustered together, and the most favorable conformation was represented by the lowest free energy of binding $(\Delta \mathrm{G})$ and the lowest inhibition constant (Ki). The lowest binding energy conformation of the compounds was selected and evaluated for molecular interactions with their receptors using LigPlot $+\mathrm{v}$ 1.4.5 (Laskowski and Swindells, 2011). To ensure that the binding poses of the docked compounds represented favorable and valid potential binding modes, the docking parameters and methods were validated by redocking the cocrystal ligand in order to determine the ability of AutoDock to reproduce the orientation and position of the ligand observed in the crystal structure.

\section{RESULTS AND DISCUSSION}

The redocking of cocrystal ligands to their respective molecular targets exhibited an RMSD value of $<2 \AA$ between the original cocrystal ligand position and the docked poses (Figure 2). This confirmed that the ligands were closely bound to the true conformation of their targets indicating the reliability of the docking protocols and parameters.

The proceraside A, frugoside and calotropin compounds were docked against the following seven molecular targets to gain insights into their possible binding modes (Figure 3): CDK-2, CDK-6, topoisomerase I, BCL-2, VEGFR-2, telomere: G-quadruplex, and topoisomerase II. The binding energies and inhibition constants of proceraside A, frugoside and calotropinare shown in Table 2. The lowest binding poses of the best docked ligand were selected for analyses of molecular interactions between hydrogen bonds and hydrophobic interactions. The hydrogen bonds and hydrophobic interactions between the best docked ligand and molecular targets have been summarized in Table 3.

Proceraside A was docked to CDK-2 and CDK-6 with binding energies of -11.19 and $-10.53 \mathrm{kcal} / \mathrm{mol}$ and inhibition constants of -6.30 and $19.16 \mathrm{nM}$, respectively, which were considerably lower than those of their cocrystalligands. Both CDK-2 and CDK-6 belong to the core-cell cycle machinery and they exert catalytic functions when bound to cyclins. Furthermore, they play crucial roles in cell cycle regulation, apoptosis, transcription, and neuronal functions (Dai and Grant, 2003; Huwe et al., 2003). The good binding interaction of proceraside A with CDK-2 may be attributed to the formation of six hydrogen bonds via the following: the backbone $\mathrm{O}$ atom of Glu12, and Glu81; the $\mathrm{N}$ atom of Leu83 and Thr165 and hydrophobic interactions mediated through Ile10, Gly11, Gly13, Val18, Ala31, Val64, Phe80, Phe82, Lys129, Gln131, Asn132, Leu134, Val163, and Val164. Interestingly, all of the hydrogen bonds were only established through the backbone $\mathrm{O}$ and $\mathrm{N}$ atoms of the contributing residues. Similarly, proceraside interacted well with CDK-6 and this interaction was composed of five hydrogen bonds via the following: the side chain $\mathrm{O}$ atom of Asp104; the side chain $\mathrm{O}$ atom of Thr107; the side chain $\mathrm{O}$ atom and backbone $\mathrm{N}$ atom of Asp163 


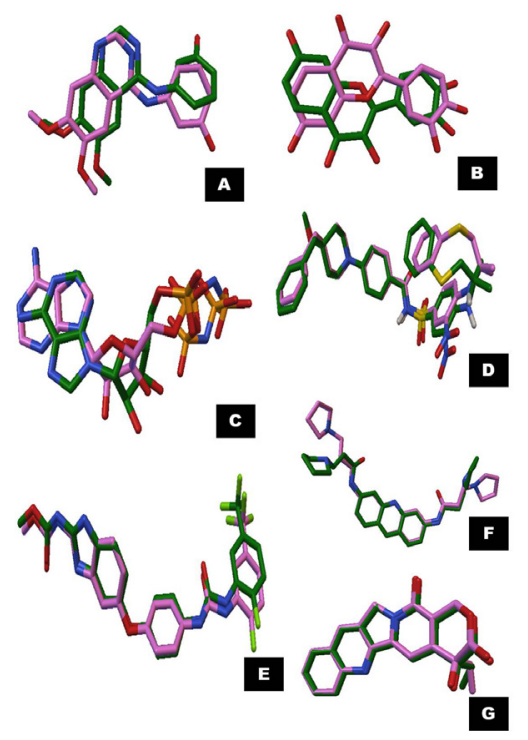

Figure 2. A.-G. Docking validation by redocking the cocrystal ligands to their corresponding molecular targets as indicated by their PDB IDs. The original conformation of each cocrystal ligands is displayed in green, stick while docked poses are represented in pink stick. The root mean square deviation (RMSD) was calculated between the

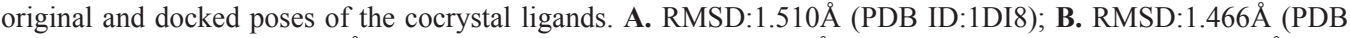

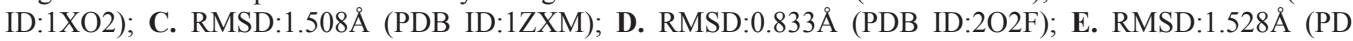

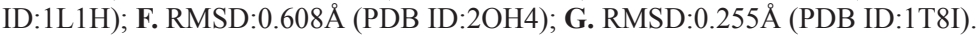

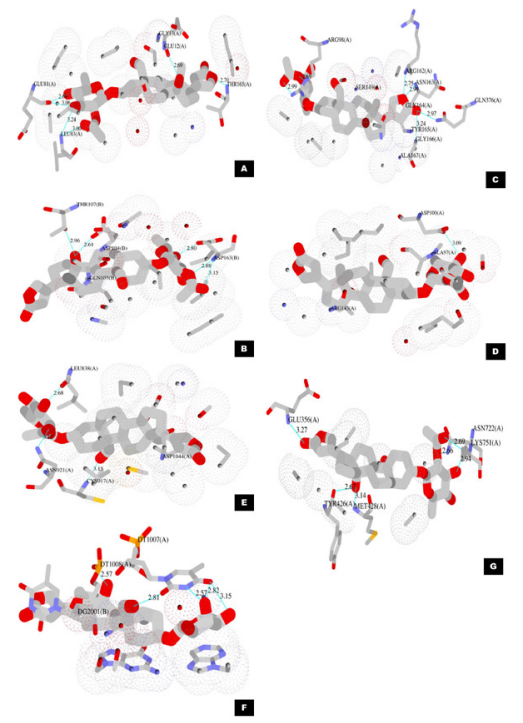

Figure 3. Molecular interaction between the best docked ligand and target proteins A. 1di8 proceraside A; B. 1xo2_proceraside; C. 1zxm_proceraside A; D. 2o2f_proceraside A; E. 2oh4_proceraside A; F. 111h_frugoside A; G. 1 t8i proceraside A. The ligands are shown with thick sticks, while the residues interacting via $\mathrm{H}$-bonds are shown as thin sticks. H-bonds are shown in cyan. The dotted surfaces correspond to the residue parts that interact with the ligand via hydrophobic interactions. 
and hydrophobic interactions via Ile19, Gly22, Val27, Lys43, Val77, Phe98, His100, Val101, Asp102, Glu103, Gln149, Asn150, Leu152, and Ala162.

Table 2. Binding energies and inhibition constants of selected Calotropis procera-derived compounds that were docked against molecular targets.

\begin{tabular}{|c|c|c|c|c|c|c|c|c|c|c|c|c|c|c|}
\hline \multirow[t]{3}{*}{ Compounds } & \multicolumn{14}{|c|}{ Drug targets (PDB Entries) } \\
\hline & \multicolumn{2}{|c|}{ CDK-2 (1DI8) } & \multicolumn{2}{|c|}{ CDK-6(1XO2) } & \multicolumn{2}{|c|}{ Topoisomerase II (1ZXM) } & \multicolumn{2}{|c|}{$\begin{array}{l}\text { BCL-2 (2O2F) } \\
\text { BCL }\end{array}$} & \multicolumn{2}{|c|}{ VEGFR-2 (2OH4) } & \multicolumn{2}{|c|}{ Telomere: G-quadruplex (ILIH) } & \multicolumn{2}{|c|}{ Topoisomerase I (IT8I) } \\
\hline & \begin{tabular}{c|c|}
$\mathrm{BE}$ \\
$(\mathrm{kcal} / \mathrm{mol})$
\end{tabular} & $\begin{array}{c}\mathrm{Ki} \\
(\mathrm{nM})\end{array}$ & \begin{tabular}{c|c|}
$\mathrm{BE}$ \\
$(\mathrm{kcal} / \mathrm{mol})$
\end{tabular} & $\begin{array}{c}\mathrm{Ki} \\
(\mathrm{nM})\end{array}$ & $\begin{array}{c}\mathrm{BE} \\
(\mathrm{kcal} / \mathrm{mol})\end{array}$ & $\mathrm{Ki}(\mathrm{nM})$ & $\begin{array}{c}\mathrm{BE} \\
(\mathrm{kcal} / \mathrm{mol})\end{array}$ & Ki (nM) & $\begin{array}{c}\mathrm{BE} \\
(\mathrm{kcal} / \mathrm{mol})\end{array}$ & $\begin{array}{c}\mathrm{Ki} \\
(\mathrm{nM})\end{array}$ & $\begin{array}{c}\mathrm{BE} \\
(\mathrm{kcal} / \mathrm{mol})\end{array}$ & $\begin{array}{c}\mathrm{Ki} \\
(\mathrm{nM})\end{array}$ & $\begin{array}{c}\mathrm{BE} \\
(\mathrm{kcal} / \mathrm{mol})\end{array}$ & $\begin{array}{c}\mathrm{Ki} \\
(\mathrm{nM})\end{array}$ \\
\hline roceraside $\mathrm{A}$ & -11.19 & 6.30 & -10.53 & 19.16 & -11.09 & & -8.97 & 264 & -11.31 & 5.14 & -7.11 & 6120.00 & -11.69 & 2.69 \\
\hline & & 103.99 & & 52.20 & & & & & -9.46 & & & 1000.00 & & 36.94 \\
\hline Calotrof & & 37.95 & -8.32 & 800.24 & -8.20 & 979.05 & -8.44 & & -8.64 & 464.07 & & $30,820.00$ & -10.66 & 15.41 \\
\hline Cocrystal li & -8.04 & 1270.00 & -8.26 & 882.71 & -11.11 & 7.24 & -11.01 & 8.56 & -12.46 & 0.738 & -11.97 & 1.68 & -10.75 & 13.23 \\
\hline
\end{tabular}

Numbers in bold indicate compound best docked with target protein. $\mathrm{BE}=$ estimated free energy of binding $[\mathrm{BE}=$ final intermolecular energy + final total internal energy + torsional free energy - unbound system's energy], where the final intermolecular energy $=\mathrm{vdW}+$ Hbond + desolv energy + electrostatic energy; $\mathrm{Ki}=$ estimated inhibition constant [temperature $=298.15 \mathrm{~K}]$.

Table 3. LigPlot + results of molecular interactions between the best docked ligand and molecular targets.

\begin{tabular}{|c|c|c|c|}
\hline \multirow[t]{2}{*}{ Protein ligand complex } & \multicolumn{2}{|c|}{ Hydrogen bonds } & \multirow[t]{2}{*}{ Hydrophobic interactions } \\
\hline & Interacting atoms & Bond length $(\AA)$ & \\
\hline \multirow[t]{6}{*}{ 1di8_proceraside $\mathrm{A}$} & $\mathrm{O} 3 \ldots \ldots . . \mathrm{O}(\mathrm{GLU} 12)$ & 2.69 & \multirow{6}{*}{$\begin{array}{l}\text { ILE10, GLY11, GLY13, VAL18, ALA31, VAL64, PHE80, PHE82, LYS129, GLN131, ASN132, LEU134, VAL163, } \\
\text { VAL164 }\end{array}$} \\
\hline & O7......O(GLU81) & 3.06 & \\
\hline & O6...... O(GLU81) & 2.69 & \\
\hline & O9........(LEU83) & 3.00 & \\
\hline & 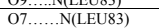 & 3.24 & \\
\hline & O1.......N(THR165) & 2.70 & \\
\hline \multirow[t]{5}{*}{ 1x02_proceraside $\mathrm{A}$} & O3...... D 1(ASP104) & 2.61 & \multirow{5}{*}{$\begin{array}{l}\text { ILE19, GLY22, VAL27, LYS43, VAL77, PHE98, HIS100, VAL101, ASP102, GLU103, GLN149, ASN150, LEU152, } \\
\text { ALA162 }\end{array}$} \\
\hline & 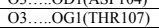 & $\frac{2.01}{2.96}$ & \\
\hline & O6....OD2(ASP163) & 2.80 & \\
\hline & O8...N(ASP163) & 2.88 & \\
\hline & O9......N(ASP163) & 3.15 & \\
\hline \multirow[t]{6}{*}{ 1zxm_proceraside A } & O9....NH2(ARG98) & 2.99 & \multirow[t]{6}{*}{ ASN81, ILE125, PRO126, VAL137, ILE141, SER148, SER149, ASN150, GLY161, GLY164, TYR165, ALA167 } \\
\hline & O8.....NE(ARG98) & 2.69 & \\
\hline & O1.....N(ARG162) & 2.75 & \\
\hline & 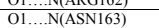 & 2.99 & \\
\hline & 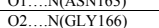 & 3.24 & \\
\hline & O2....NE2(GLN376) & 2.97 & \\
\hline $202 \mathrm{f}$ proceraside $\mathrm{A}$ & O6....OD2(ASP100) & 3.09 & \multirow{4}{*}{$\begin{array}{l}\text { THR93, GLN96, ALA97, PHE101, TYR105, LEU134, GLY142, ARG143, VAL145, ALA146, TYR199 } \\
\text { VAL846, ALA864, LYS866, GLU883, LEU887, VAL897, VAL914, GLU915, PHE916, LEU1033, CYS1043, } \\
\text { ASP1044, PHE1045 }\end{array}$} \\
\hline \multirow[t]{3}{*}{ 20h4_proceraside A } & O7.....(LEU838) & $\frac{3.07}{2.68}$ & \\
\hline & O4....N(CYS917) & 3.15 & \\
\hline & \begin{tabular}{|l} 
O9.....ND2(ASN921) \\
\end{tabular} & 3.12 & \\
\hline \multirow{5}{*}{ 111h_frugoside A } & O5....O4(DT1007) & 3.15 & \multirow{5}{*}{ DT1006, DG1009, DG2001, DG2012 } \\
\hline & O6....04(DT1007) & 2.82 & \\
\hline & O6....N3(DT1007) & 2.57 & \\
\hline & O9.....O2(DT1007) & 2.81 & \\
\hline & \begin{tabular}{|l} 
O $3 \ldots . . . .0 P 1(\mathrm{DT} 1008)$ \\
\end{tabular} & $\frac{2.81}{2.57}$ & \\
\hline \multirow[t]{6}{*}{ 1t8i_proceraside $\mathrm{A}$} & $\mathrm{O} 2 \ldots . . \mathrm{N}(\mathrm{GLU} 356)$ & 3.27 & \multirow[t]{6}{*}{ ALA351, ASN352, TRP416, ILE427, LEU429, PRO431, LYS436, LEU721 } \\
\hline & $\begin{array}{l}03 \ldots . . . \mathrm{O}(\mathrm{TYR} 426) \\
\mathrm{O}\end{array}$ & $\frac{3.27}{2.67}$ & \\
\hline & 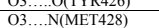 & $\frac{2.07}{3.14}$ & \\
\hline & O8....ND2(ASN722) & 2.66 & \\
\hline & $\begin{array}{l}07 \ldots . . N Z(L Y S 751) \\
\end{array}$ & $\frac{2.90}{2.94}$ & \\
\hline & $\begin{array}{l}09 . . . . \mathrm{NZ}(\text { LYS751) } \\
\end{array}$ & 2.69 & \\
\hline
\end{tabular}

Proceraside A showed an impressive binding energy of $-11.69 \mathrm{kcal} / \mathrm{mol}$ and an inhibition constant of $2.69 \mathrm{nM}$ with topoisomerase I, which was notably lower than that of the cocrystal ligand. However, with topoisomerase II, its binding energy $(-1.09 \mathrm{kcal} / \mathrm{mol})$ and inhibition constant $(7.40 \mathrm{nM})$ values were comparable to those of the cocrystal ligand. DNA topoisomerases I and II have both been implicated in cell survival and they play critical roles in DNA metabolism and structure (Phosrithong and Ungwitayatorn, 2010). Proceraside A showed the best interaction with topoisomerases I, which involved six hydrogen bonds formed via the following: the backbone $\mathrm{N}$ atom of Glu356, the backbone $\mathrm{O}$ atom of Tyr426; the backbone N atom of Met428; the side chain N atoms of Asn722 and Lys751 and the hydrophobic interactions via Ala351, Asn352, Trp416, Ile427, Leu429, Pro431, Lys436, and Leu721. Proceraside A also displayed a good interaction with topoisomerase I through establishment of six hydrogen bonds via the following: the side chain $\mathrm{N}$ atom of $\mathrm{Arg} 98$; the 
backbone N atom of Arg162, Asn163 and Gly166 and the side chain N atom of Gln376; and the hydrophobic interactions via Asn81, Ile125, Pro126, Val137, Ile141, Ser148, Ser149, Asn150, Gly161, Gly164, Tyr165, and Ala167.

Proceraside A exhibited binding energy of $-8.97 \mathrm{kcal} / \mathrm{mol}$ and an inhibition constant of $264.15 \mathrm{nM}$ with BCL-2, which was significantly higher than that of the cocrystal ligand. BCL-2 is an anti-apoptotic oncoprotein that affects neoplastic cell proliferation by preventing cell death (Reed, 1994). However, proceraside, did not exhibit a favorable interaction with BCL-2, which may be due to the fact that it was only able to establish one hydrogen bond with the side chain $\mathrm{O}$ atom of Asp100. Furthermore, the major interaction was contributed to hydrophobic interactions via Thr93, Gln96, Ala97, Phe101, Tyr105, Leu134, Gly142, Arg143, Val145, Ala146, and Tyr199.

Proceraside A docked with VEGFR-2 with a binding energy of $-11.31 \mathrm{kcal} / \mathrm{mol}$ and an inhibition constant of $5.14 \mathrm{nM}$, which was considerably higher than the cocrystal ligand. VEGFR-2 is a cell surface receptor for VEGF that is highly expressed on vascular endothelial cells, which can modulate vascular endothelial survival, proliferation, migration, and the formation of vascular tubes (Veikkola et al., 2000). Proceraside A was able to establish three hydrogen bonds via the following: the backbone $\mathrm{O}$ atom of Leu838; the backbone $\mathrm{N}$ atom of Cys917; the side chain $\mathrm{N}$ atom of Asn921, and the hydrophobic interactions via Val846, Ala864, Lys866, Glu883, Leu887, Val897, Va1914, Glu915, Phe916, Leu1033, Cys1043, Asp1044, and Phe1045.

However, proceraside A did not exhibit adequate binding energy with telomere: G-quadruplex, and this is likely to be because of its inability to establish an adequate number of hydrogen bonds and hydrophobic interactions. We found that frugoside best docked with telomere: G-quadruplex with a binding energy of $-8.18 \mathrm{kcal} / \mathrm{mol}$ and an inhibition constant of $1000 \mathrm{nM}$, and these values were significantly higher than those of the cocrystal ligand. Telomeres are highly complex nucleo-protein structures at the end of eukaryotic chromosomes that influence the proliferative capacity of cells. Mammalian telomeric DNA is composed of G-rich tandem repeats (TTAGGG) . Moreover, the bulk of telomeric DNA is double-stranded, but the extreme terminus consists of 3'G-rich single-stranded overhangs composed of several hundred bases that act as substrates to which telomeric repeats are added by the telomerase enzyme (Henderson and Blackburn, 1989; Satyanarayana et al., 2004). Although, frugoside was able to establish five hydrogen bonds via the $\mathrm{O}$ and $\mathrm{N}$ atoms of the DT1007 pyrimidine ring and the $\mathrm{O}$ atom of the DT1008 phosphate backbone, it exhibited only fewer hydrophobic interactions via DT1006, DG1009, DG2001, and DG2012.

In conclusion, the molecular docking analysis of proceraside $\mathrm{A}$, frugoside, and calotropin with macromolecules involved in the cell cycle and DNA replication identified proceraside $\mathrm{A}$ as the best docked ligand, and it exhibited good interactions with CDK-2, CDK-6, topoisomerase I, BCL-2, VEGFR-2, and topoisomerase II. Proceraside A showed the lowest binding energies and lowest inhibition constants, and it displayed a greater number of hydrogen bonds and hydrophobic interactions with the molecular targets, which indicated its higher binding affinity towards the selected molecular targets. The present study may aid in target fishing for proceraside $\mathrm{A}$ and studies of its binding mode might provide a reasonable explanation for its experimentally proven anti-tumor properties. Furthermore, this study can be extended to analyze structure-activity relationships to derive more potent target-specific inhibitors. 


\section{Conflicts of interest}

The authors declare no conflict of interest.

\section{ACKNOWLEDGMENTS}

The authors would like to extend their sincere appreciation to the Deanship of Scientific Research at King Saud University for funding this research through the Research Group Project (\#RGP-195).

\section{REFERENCES}

Dai Y and Grant S (2003). Cyclin-dependent kinase inhibitors. Curr. Opin. Pharmacol. 3: 362-370. http://dx.doi. org/10.1016/S1471-4892(03)00079-1

Gan DD, Macaluso M, Cinti C, Khalili K, et al. (2003). How does a normal human cell become a cancer cell? J. Exp. Clin. Cancer Res.22: 509-516.

Greenlee RT, Murray T, Bolden S and Wingo PA (2000). Cancer statistics, 2000.CA Cancer J. Clin.50: 7-33.http://dx.doi. org/10.3322/canjclin.50.1.7

Halgren TA (1996). Merck molecular force field. I. Basis, form, scope, parameterization, and performance of MMFF94. J. Comput. Chem. 17: 490-519. http://dx.doi.org/10.1002/(SICI)1096-987X(199604)17:5/6<490::AID$\mathrm{JCC} 1>3.0 . \mathrm{CO} ; 2-\mathrm{P}$

Henderson ER and Blackburn EH (1989). An overhanging 3' terminus is a conserved feature of telomeres. Mol. Cell. Biol. 9: 345-348. http://dx.doi.org/10.1128/MCB.9.1.345

Huwe A, Mazitschek R and Giannis A (2003). Small molecules as inhibitors of cyclin-dependent kinases. Angew. Chem. Int. Ed. Engl. 42: 2122-2138. http://dx.doi.org/10.1002/anie.200200540

Ibrahim SRM, Mohamed GA, Shaala LA, Moreno L, et al. (2014). Proceraside A, a new cardiac glycoside from the root barks of Calotropis procera with in vitro anticancer effects. Nat. Prod. Res. 28: 1322-1327. http://dx.doi.org/10.10 $\underline{80 / 14786419.2014 .901323}$

Laskowski RA and Swindells MB (2011). LigPlot+: multiple ligand-protein interaction diagrams for drug discovery. $J$. Chem. Inf. Model. 51: 2778-2786. http://dx.doi.org/10.1021/ci200227u

Morris GM, Huey R, Lindstrom W, Sanner MF, et al. (2009). AutoDock4 and AutoDockTools4: Automated docking with selective receptor flexibility. J. Comput. Chem. 30: 2785-2791.http://dx.doi.org/10.1002/jcc.21256

Phosrithong N and Ungwitayatorn J (2010). Molecular docking study on anticancer activity of plant-derived natural products. Med. Chem. Res. 19: 817-835. http://dx.doi.org/10.1007/s00044-009-9233-5

Reed JC (1994). Bcl-2 and the regulation of programmed cell death. J. Cell Biol. 124: 1-6. http://dx.doi.org/10.1083/ jcb.124.1.1

Satyanarayana A, Manns MP and Rudolph KL (2004). Telomeres, telomerase and cancer: an endless search to target the ends. Cell Cycle 3: 1138-1150. http://dx.doi.org/10.4161/cc.3.9.1152

Siegel RL, Miller KD and Jemal A (2015). Cancer statistics, 2015. CA Cancer J. Clin. 65: 5-29. http://dx.doi.org/10.3322/ caac. 21254

Stark JL and Powers R (2012). Application of NMR and molecular docking in structure-based drug discovery. In: NMR of proteins and small biomolecules (Zhu G, ed.). Springer, Berlin Heidelberg, Top. Curr. Chem. 326: 1-34.

Veikkola T, Karkkainen M, Claesson-Welsh L and Alitalo K (2000). Regulation of angiogenesis via vascular endothelial growth factor receptors. Cancer Res.60: 203-212. 